\title{
Designing Fuzzy Model for Applying the Linear Method for Enhancing the Edges of the Satellite Images
}

\author{
Omar M.A. Al-Dewajy \\ College of Computer Science and Mathematics \\ University of Mosul, Iraq
}

\section{Received on: 23/04/2008}

\section{ABSTRACT}

This research aims at designing Fuzzy model for applying the Linear Method for Enhancing the Edges of the Satellite Images. The researcher depended on some information about the satellite image like (brightness value, gray level, lines...etc) and connect them with the fuzzy logic for producing fuzzy system dealing with the contents of the satellite image.

The fuzzy system designed by scaling the two inputs (Brightness Value) and (Gray Level) and producing single output (Result B.V.), and it is built with (4 rules) that evaluate the performance of the system. The system was programmed by (Matlab 7) and works under WindowsXP.

Keywords: fuzzy system, fuzzy model, satellite image.

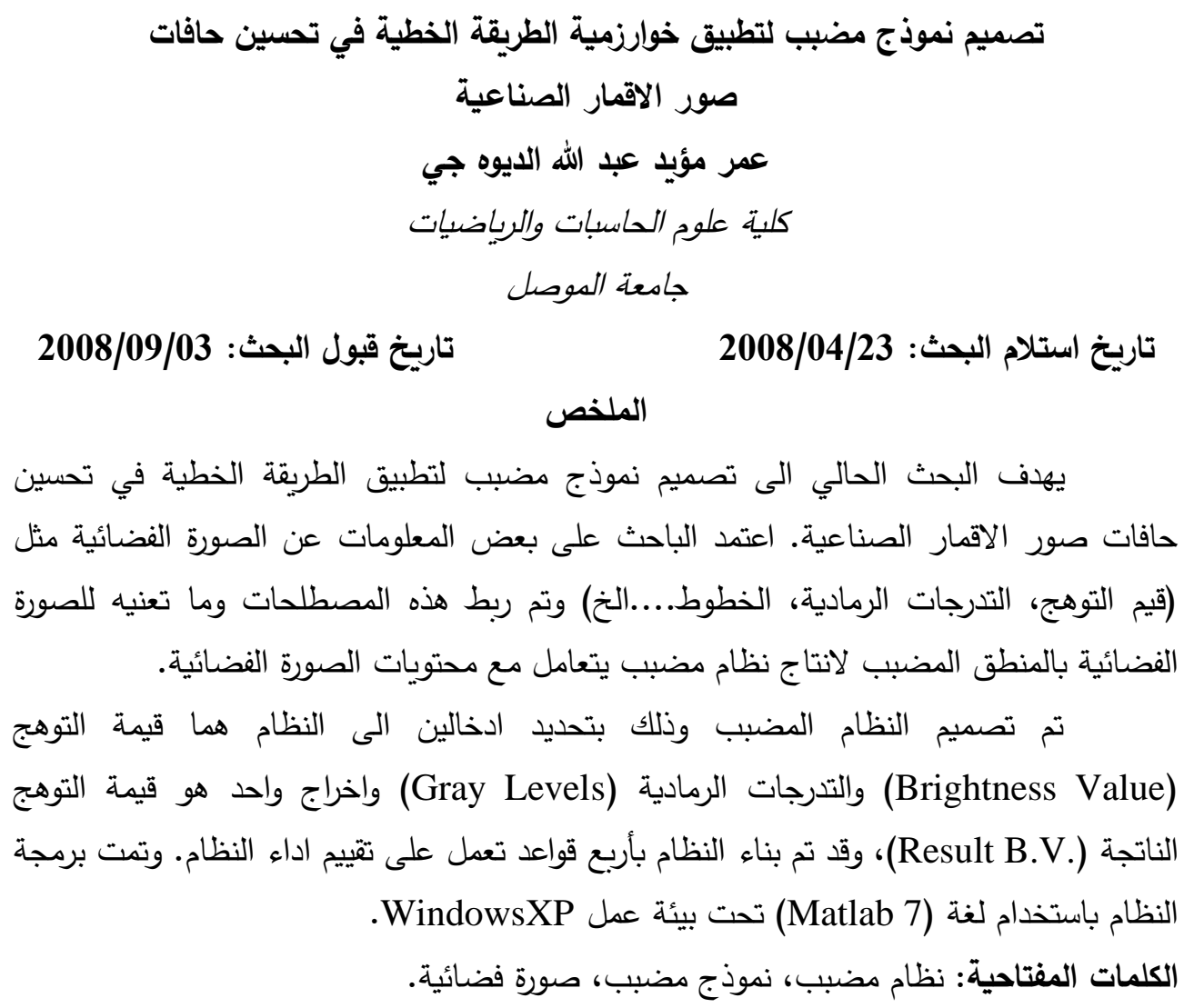




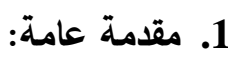

توفر الاقمار الصناعية اثناء دورانها حول الارض معلومات مهمة عن سطح الكرة

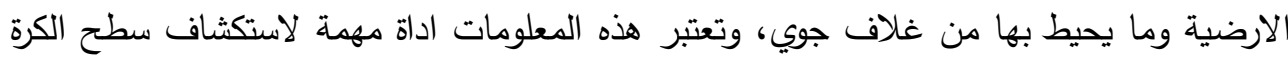

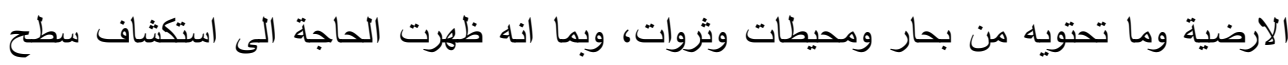

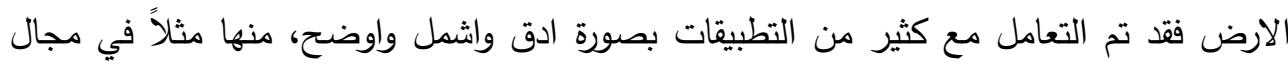

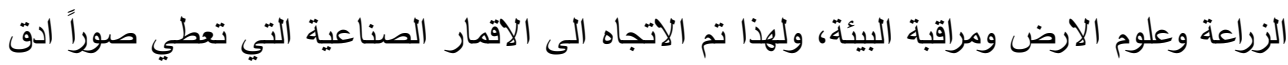
واوضح يمكن من خلالها التوصل الى نتائج في مختلف التطبيقات.

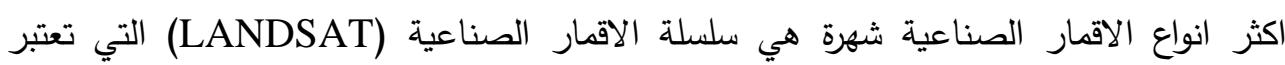

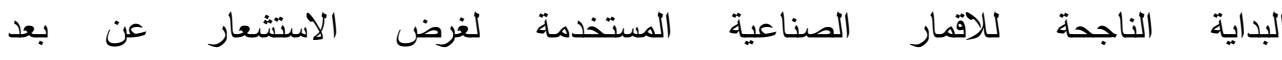
(Remote Sensing)

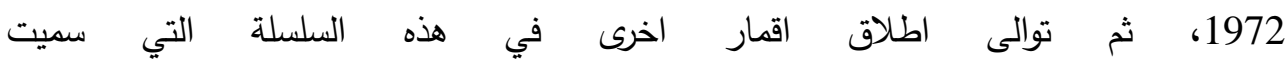

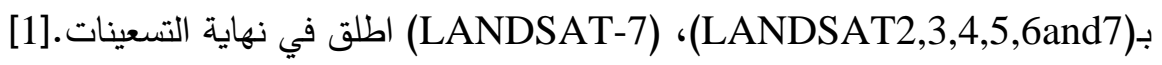
تم اعتماد فكرة ربط نموذج المنطق المضبب مع بيانات صور الاقمار الصناعية وذلك لتحديد طبيعة الصورة الفضائية. 2. اهداف البحث:

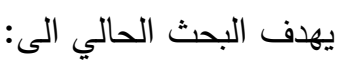

1- تصميم نموذج مضبب يعمل على توضيح معالم الصورة الفضائية بما تحتويه من خطوط

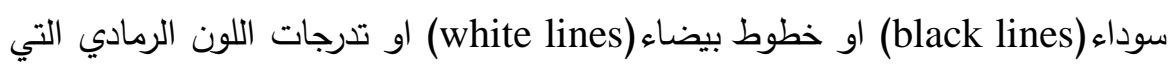
تمثل المساحات الواسعة في الصورة الفضائية. 2- امكانية معالجة بيانات الصورة الفضائية بعد تحليلها وتقسيمها الى مجموعة من المناطق ذات المساحات الواسعة التي تمثل الصحاري والبحار والتي تكون متقاربة القيم في الصورة الفضائية ومناطق اخرى مزدحمة والتي تمثل المدن او غير ذلك.

3. تحسين صور الاقمار الصناعية:

يمكن تعريف عملية التحسين التي تجري على صور الاقمار الصناعية بما تحتويه من

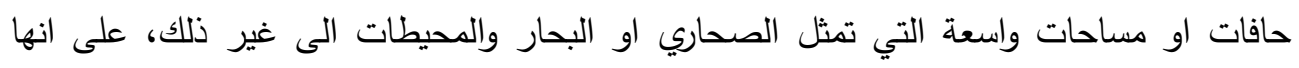

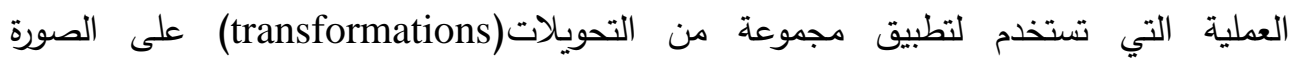


الدخلة(input satellite image) للحصول على صورة تتمتع بوضوح اكثر وتفاصيل ادق

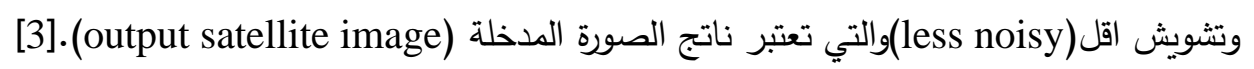

4. الماسحات الضوئية (Scanners):

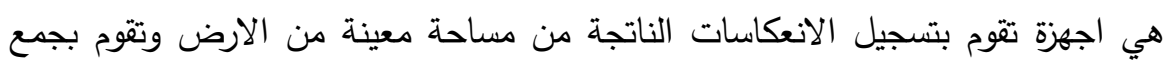
بيانات مهمة عن سطح الكرة الارضية وما تحتويه من مصادر وثروات والتي يستخدمها العلماء

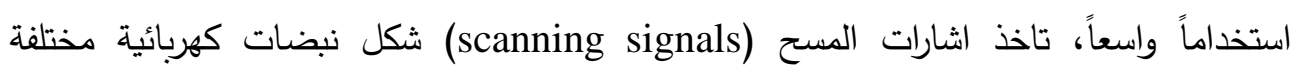
ومتتابعة يتم ارسالها الى الكومبيوتر الذي يكون قادرا على بناء صورة الصفحة بكل ماتحتويه من الفات

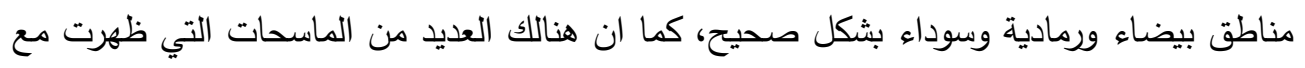

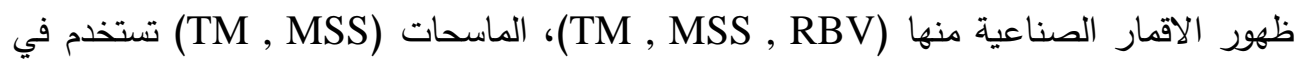

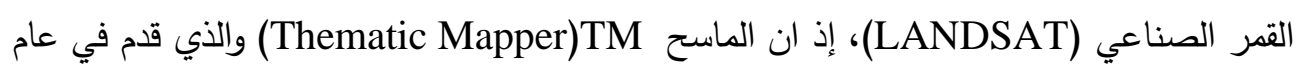
1982 يقوم بتسجيل الانعكاسات ويقوم بجمع الخيالات متعددة الاطياف، اما الماسح (Multi Spectral Scanner)

5. - 5موذج المنطق المضبب: هو تقنية تتمتع بقدرة عالية في ايجاد الحلول للمشاكل المختلفة بما في ذلك الاكاديمية منها

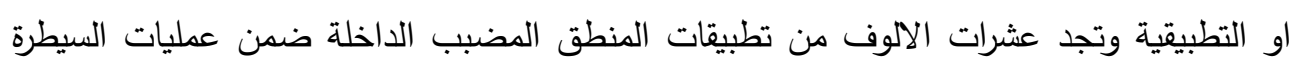

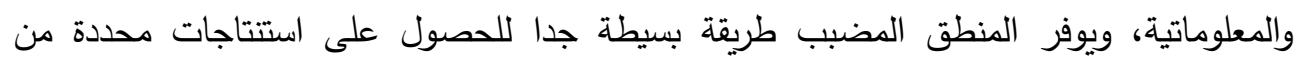

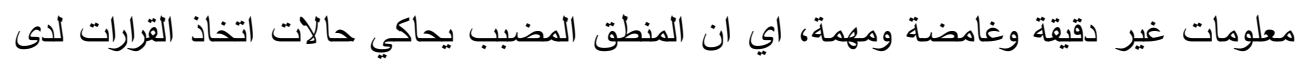

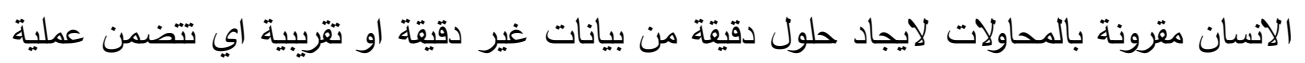
تثخيص للنظام التي يقصد بها عملية تحديد نموذج رياضي لايناميكية النظام باستخدام البيانات

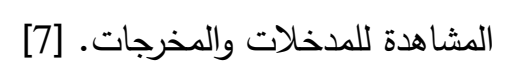

ويهذف المنطق المضبب الذي اكتشفه في الستينات العالم لطفي زاده الى توفير الدوال والاحكام الرياضية التي تتيح ايضا طرائق حساب القيم الوسطى بين الحقيقة المطلقة التي تراوح بين

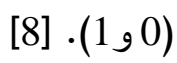

ويخل المنطق المضبب في مجالات عديدة منها الذكاء الاصطناعي، والهندسة، وبحوث

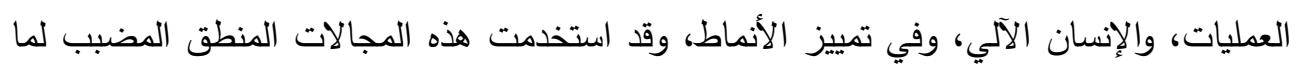

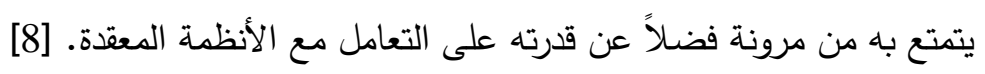


6. ميزات المنطق المضبب:

ازداد الاهتمام بالمنطق المضبب في السنوات الاخيرة مما ادى الى قبوله واستخدامه في الحقول الاكاديمية والتطبيقية على حد سواء وذلك بسب مميزاته مقارنة مع الطرائق الاخرى، ومن لهن

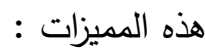
1- منهج التصميم بديل من ناحية البساطة والسرعة وذلك من خلال:

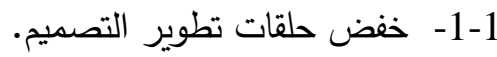
2-1-2 - 2-1 تبسيط تعقيدات التصميم. 3-1 - 3- تطوير زمن التسويق. 2- حلول بديلة افضل لنظم التحكم وذلك من خلال : 1-2 - - تطوير أداء نظام التحكم. 2-2-2 - تبسيط عمليات التنفيذ.

2-2-2 خفض كلفة الاجزاء المادية. [2]

يمكن تحديد خصائص او مميزات اي تصميم من خلال تحليل المجال الذي يهنا في المسألة إلى مناطق مضببة وذلك يتم إما عن طريق استخدام نموذج رياضي مبسط في كل منطقة أو عن طريق إنشاء ربط مباشر بين المناطق الخاصة في مجال مدخلات النموذج بالمناطق المقابلة

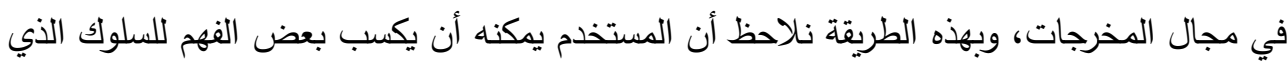

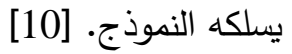

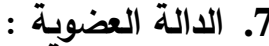

A هي دالة رياضية تعرف درجة عضوية كل عنصر في المجموعة المضببة ـ فاذا كانت هي مجموعة مضببة في المجموعة الثاملة X ، فان دالة عضوية العنصر X في X للمجموعة A

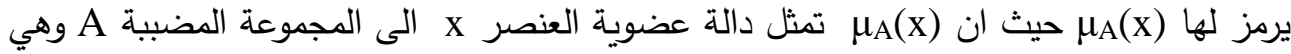
دالة رياضية تحقق الشرط الاتي[9] :-

$0<=\mu_{\mathrm{A}}(\mathrm{x})<=1$

كما أن هنالك مجموعة من الأسئلة يجب أن يتم التعامل معها عند استخدام الدوال العضوية وهي:

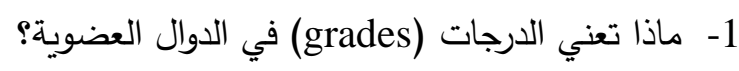
2- 2 - 2 - كيف يتم قياسها؟ 
3- ما ما العمليات أو التطبيقات المطلوب تحديدها للدالة العضوية؟

إن ابسط أنواع دوال العضوية يمكن تكوينه باستخدام الخطوط المستقيمة ومن هذه الدوال البسيطة الدالة العضوية المثلثة (Triangular membership function ) التي يطلق عليها (Trimf)

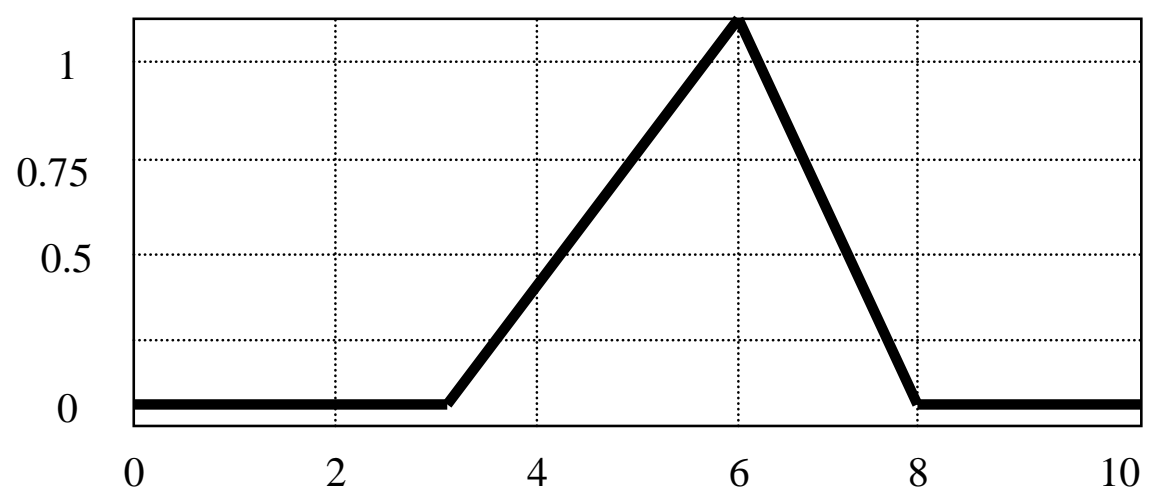

Trimf. $\mathrm{P}=\left[\begin{array}{lll}3 & 6 & 8\end{array}\right]$

\section{trimf}

Triangular الثكل(1) دالة عضوية من نوع

$$
8
$$

إن هيكلية النموذج المناسبة يتم تحديدها باستخدام معرفة مسبقة عن النظام ومقدار الخطأ

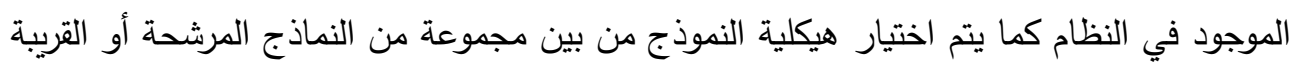

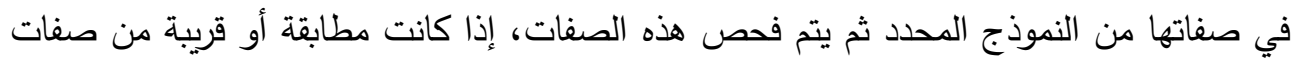
النموذج المحدد عندها يتم الاعتماد على هذا النموذج، أما إذا كانت الصفات غير ذي فائدة فئدة فيجب اجراء تغيير في هيكلية النموذج للوصول الى الهيكل القريب او المطابق لهيكل النظام المحدد]ة].

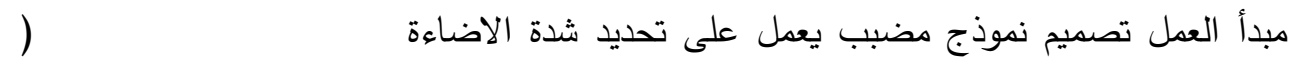
خ (Brightness Value BV خوارزمية الطريقة الخطية (Linear Method) في تطبيق عملية تحسين الحافات للصورة الفضائية التي تعتبر من احدى الطرائق البسيطة لاستخلاص الحافات من صور الاستشعار عن بعد والتي يطلق عليها (فرق التتفيذ من احثئ (nunning difference)، الصورة الاصلية تزحف نقطة ضونئية واحدة

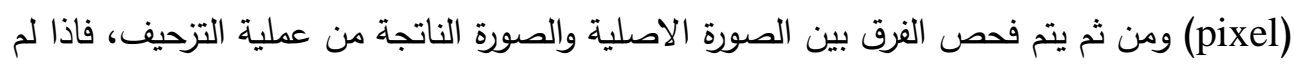
يكن هنالك فرق بين الصورتين (no difference) فلايحدث اي تاثير، اما اذا كان هنالك فرق فين 
سالب بين الصورتين فيتم تمثيلها بقيم معتمة(dark value) ومن ثم تولد خطوط سوداء( black

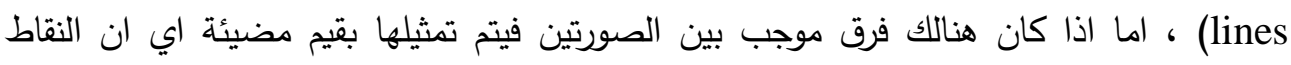

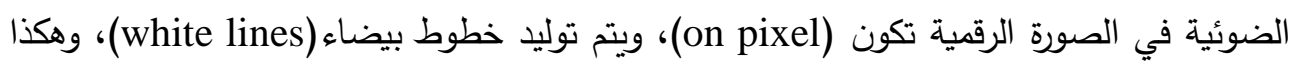
توليد خطوط بيضاء وسوداء يكون حافات لكل جزء على سطح الارض.

تم ربط نموذج المنطق المضبب مع خوارزمية الطريقة الخطية لتحسين حافات صور الاقمار الصناعية وذلك بتكوين ادخالين (2inputs) هما:

اولاً: (Bvalue) الذي يتكون من ثلاث دوال عضوية (3 Membership functions) هي: nodiff -1 التي تمثل حالة عدم وجود فرق بين الصورة الفضائية الاصلية والصورة المزحفة. negative -2 positive -3:- التي تمثل الفرق الموجب بين الصورتين ومن ثم يتم توليد قيم مضيئة.

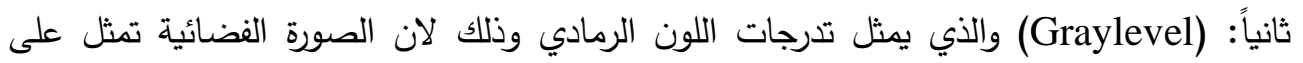
شكل رموز (ارقام) والتي تمثل بدورها تدرجات اللون الرمادي، إذ ان الخطوط البيضاء والسوداء تمثل لـاتل

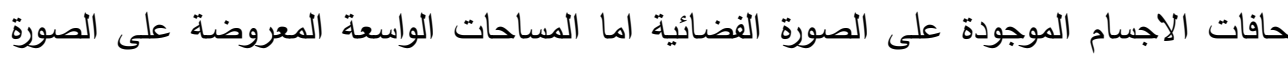

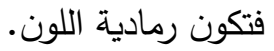
وقد تم الاعتماد على دالتين عضويتين(2 Membership functions) هما: 1 -highgray -1 والتي تمثل تدرجاً عالياً للون الرمادي، إذ ان سطح الارض يكون ذا انعكاسية عالية. lowgray -2:- والتي تمثل تدرجاً واطئًاً للون الرمادي، إذ ان سطح الارض يكون ذا انعكاسية واطئة.

اما الاخراج الذي هو ResultBV، فيعتمد على القيمة الاولية لثدة الاضاءة

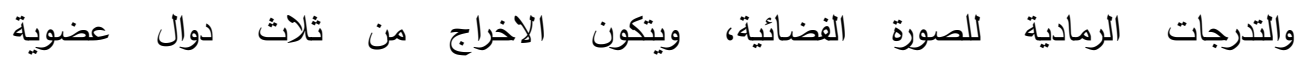
(3 Membership functions) whitelines -1 - التي تمثل الانتقال من العتمة الى الاضاءة. 2ray -2 - التي تمثل المساحات الواسعة من الصورة الفضائية. blacklines -3:- التي تمثل الانتقال من الاضاءة الى العتمة. 
لقد تم استخدام مجموعة من القواعد(Rules) والتي يتم من خلالها ربط المدخلات بالمخرجات للنظام ومن ثم تحديد النتائج، وهذه القواعد موضحة بالجدول الآتي:-

\begin{tabular}{|c|c|}
\hline Rule No. & Rule Description \\
\hline $1-$ & $\begin{array}{l}\text { If Bvalue is negative and Graylevel is lowgray Then ResultBV is } \\
\text { blacklines }\end{array}$ \\
\hline $2-$ & $\begin{array}{l}\text { If Bvalue is positive and Graylevel is highgray Then ResultBV is } \\
\text { whitelines }\end{array}$ \\
\hline $3-$ & $\begin{array}{l}\text { If Bvalue is nodiff and Graylevel is highgary Then ResultBV is } \\
\text { gray }\end{array}$ \\
\hline 4- & $\begin{array}{l}\text { If Bvalue is nodiff and Graylevel is lowgray Then ResultBV is } \\
\text { balcklines }\end{array}$ \\
\hline
\end{tabular}




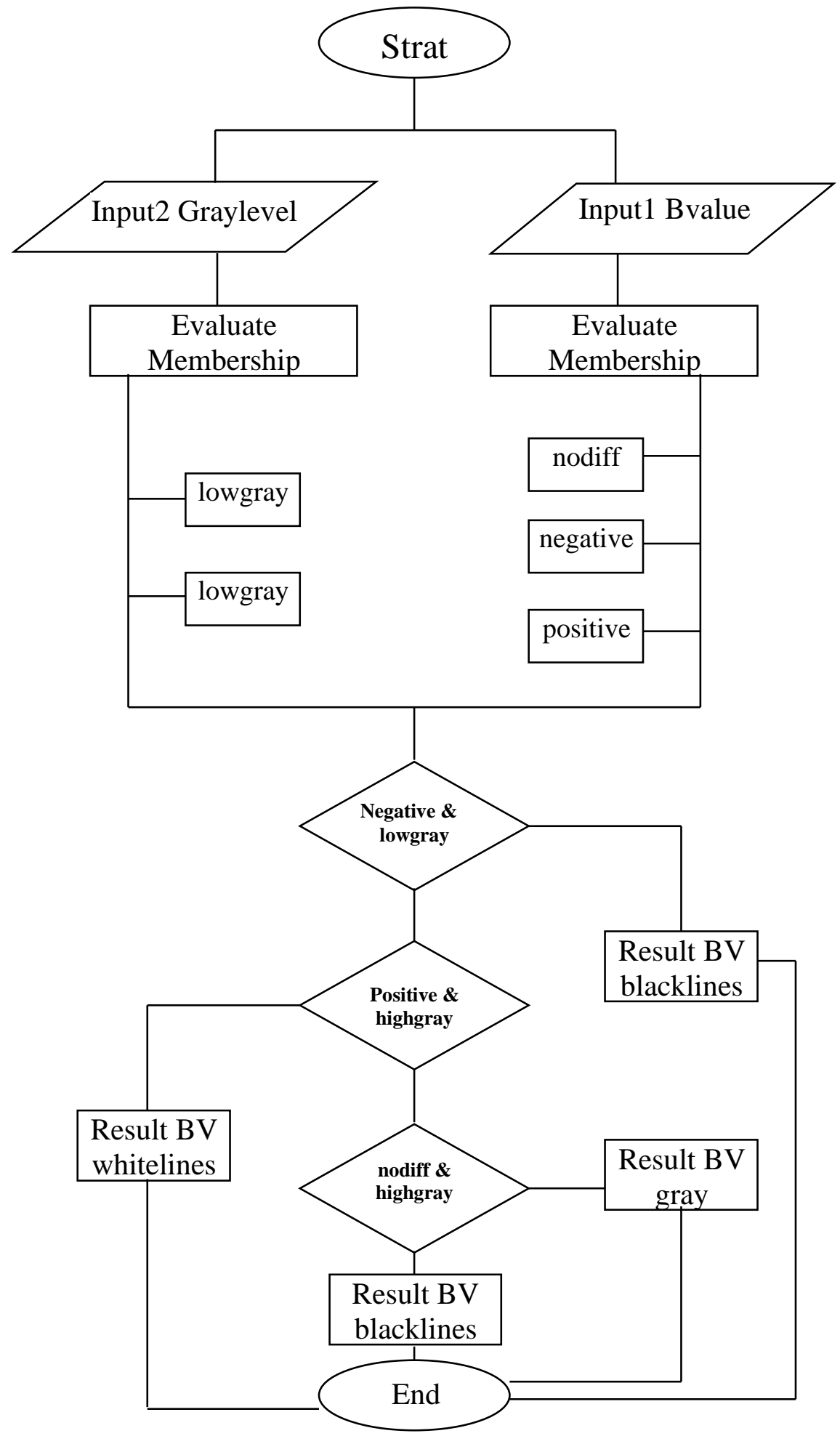


يتالف النظام من تصميم مجموعة من الواجهات الخاصة بدوال العضوية والواجهة الرئيسية

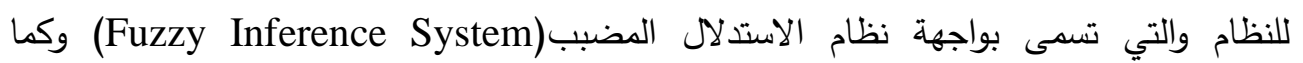

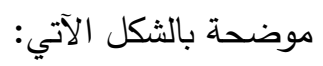

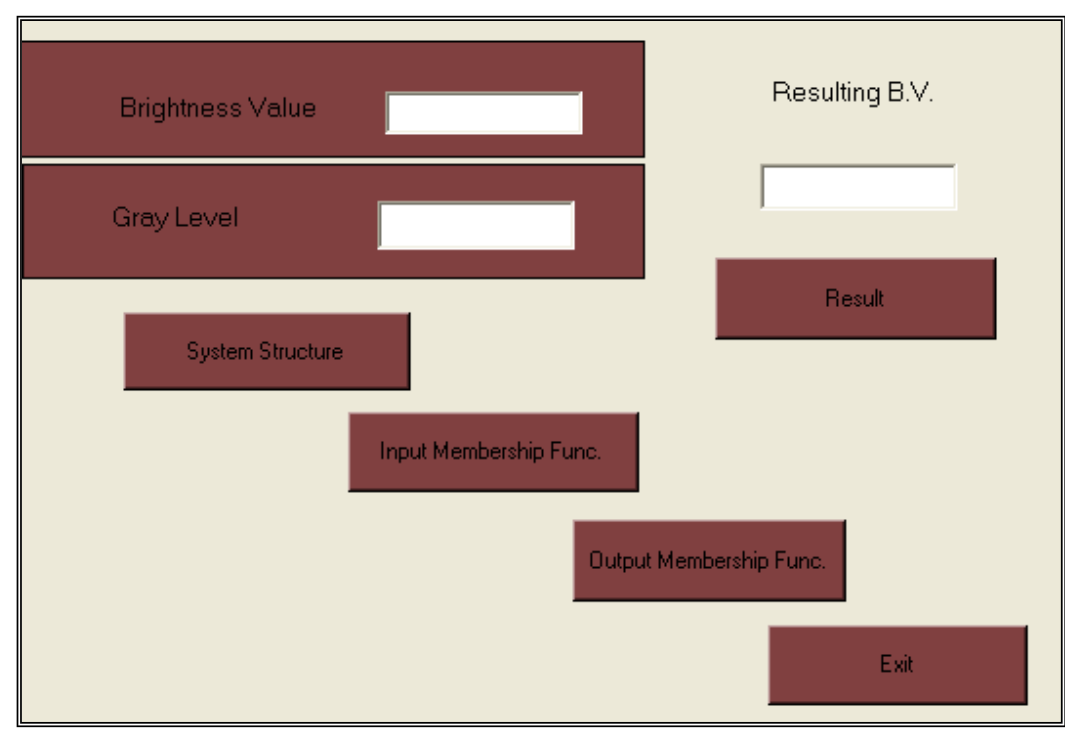

الشكل(2) يوضح الواجهة الرئيسية للنموذج المصمح

نلاحظ من الثكل اعلاه الواجهة الرئيسية للنظام والتي تتكون من:

10.1 حقل الاذخال الاول(Brightness Value) الذي يتم فيه ادخال القيمة الهثة لثدة الاضاءة. (crisp input)

10.2 حقل الادخال الثاني(Gray Level لتدرجات اللون الرمادي. (crisp input)

10.3 الاختيار الثالث (Result) الذي يقوم بعرض النتيجة النهائية لعملية التقويم(evaluation) ومن ثم يتم عرض النتيجة النهائية التي تمثل قيمة شدة الاضاءة ومن ثم يمكن تحديد هل ان هذه

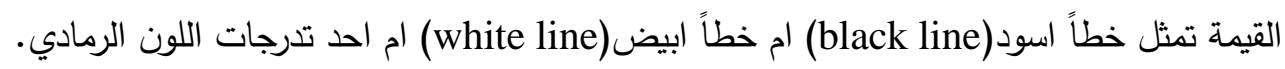

System Structure 10.4 المضبب(FIS) للنظام المصمم وكما موضح بالثكل الآتي: 


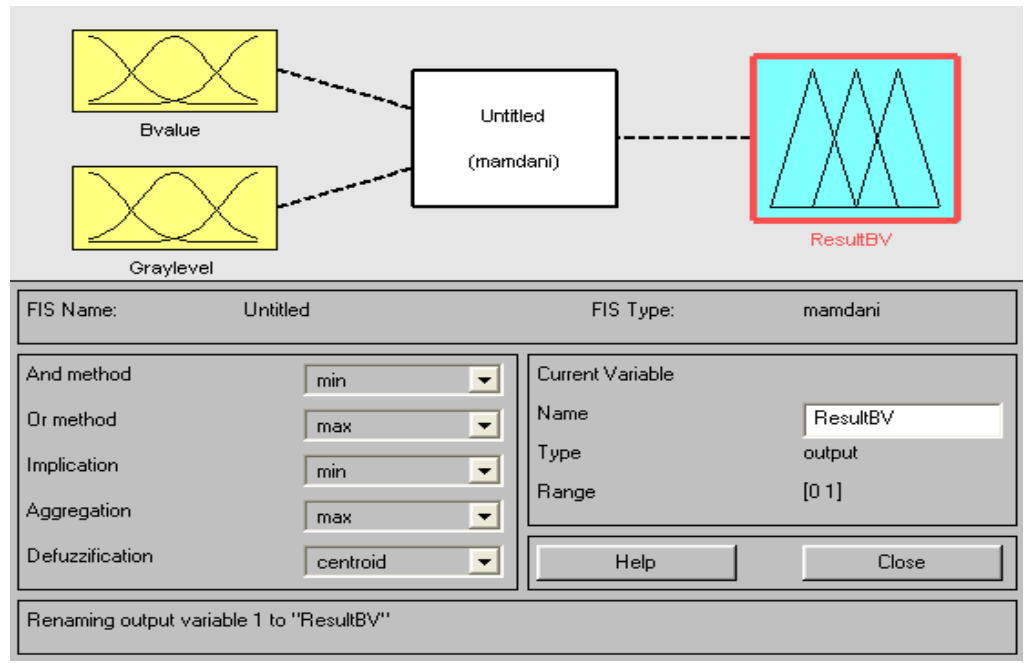

الثكل(3) يوضح هيكلية النظام

نلاحظ من الثكل اعلاه ان نموذج النظام الحالي يتكون من ادخالين (inputs) 20

واحد (single output) تربطهما مجموعة من القوانين(rules)، يتم من خلال هذه القوانين تحديد

شدة الاضاءة الناتجة(Resulting B.V) من الفرق بين الصورة الاصلية والصورة المزحفة ومن ثم تحديد الخطوط البيضاء او السوداء التي تمثل الحافات الموجودة في الصورة الفضائية والتدرجات الرمادية(gray levels) التي تمثل المساحات الواسعة الموجودة على الصورة.

10.5 للادخالين (Brightness Value) و (Gray Level) وكما موضح بالثكل الآتي: 


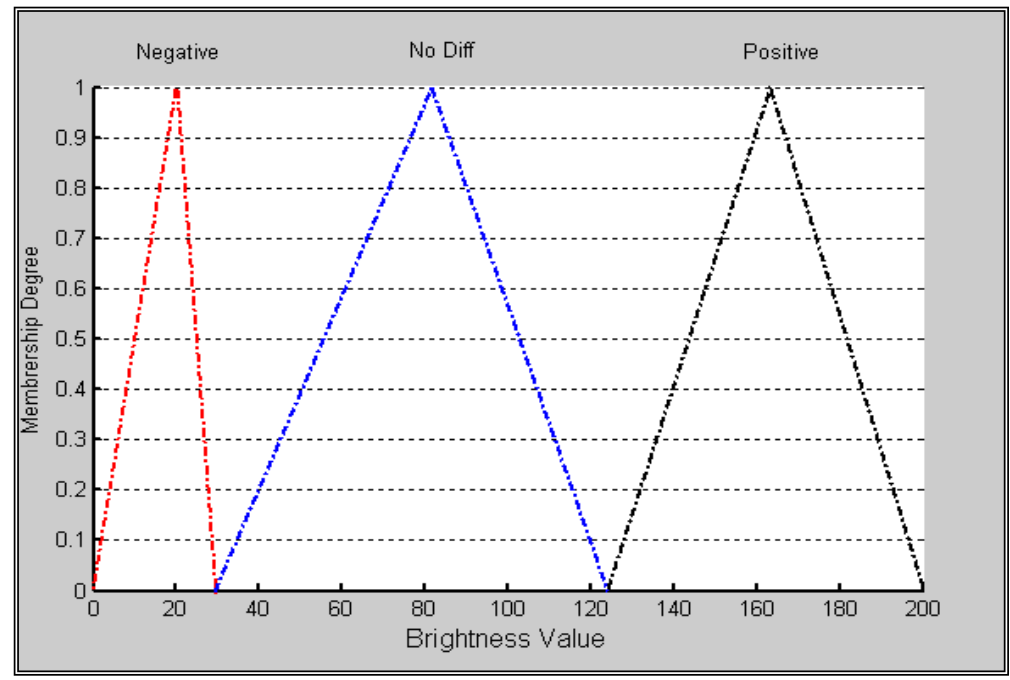

الثكل(4) دوال العضوية للادخال الاول(Brightness Value)

تم الاعتماد على تقسيم المدى لدوال العضوية الموضحة بالثكل اعلاه من خلال خوارزمية الطريقة الخطية والتي هي من احدى ابسط الطرائق لاستخلاص الحافات من صور الاقمار

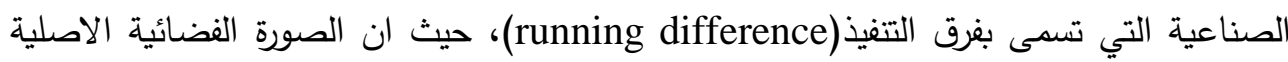
تزحف نقطة مضيئة واحدة (pixel) ومن ثم يتم فحص الفرق بين الصورتين، ويتم حساب قيمة التوهج الناتجة(Resulting Brightness Value) فاذا كان هنالك فرق سالب بين الصورتين فيتم تعويض احدى القيم المحصورة بين (30-0)، اما اذا لم يكن هنالك فرق بين الصورتين فيتم تعويض القيم المحصورة بين (127-30)، واذا كان هنالك فرق موجب بين الصورتين فيتم تعويض القيم المحصورة بين (200-127). وكما موضح بالخطوات الآتية:

$B V i, j=B V i, j-B v i-1, j$

If $\mathrm{BVi}, \mathrm{j}=-1$

Then resultingBVi,j=x where $0<=\mathrm{x}<=30$

If $\mathrm{BVi}, \mathrm{j}=0$

Then resultingBVi,j=x where $31<=\mathrm{x}<=127$

If $\mathrm{BVi}, \mathrm{j}=1$

Then resultingBVi,j $=\mathrm{x}$ where $128<=\mathrm{x}<=200$ 
ResultingBVi,j التزحيف.

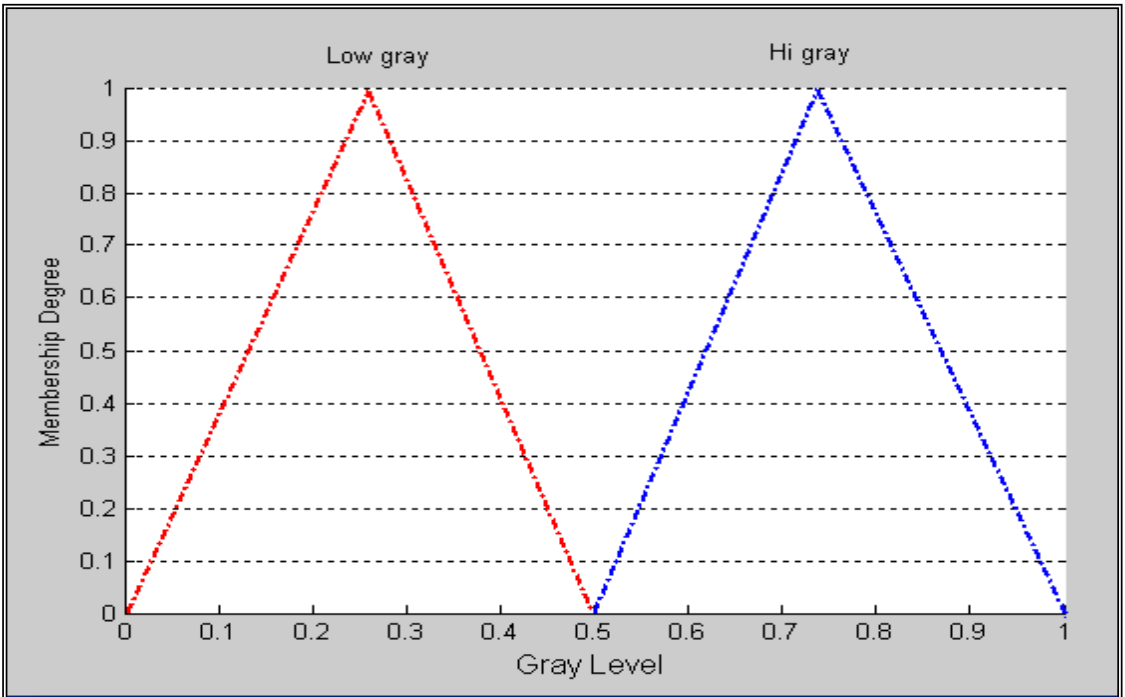

الثكل(5) دوال العضوية للادخال الثاني (Gray Level)

نلاحظ من الثكل السابق دوال العضوية للادخال الثاني الذي يتكون من دالتيين عضويتين الاولى لئ (Low gray)

لماح 10.6 للاخراج (output) الذي يمثل قيمة التوهج الناتجة من الفرق بين الصورة الفضائية الاصلية والصورة المزحفة وكما موضح بالثكل الآتي: 


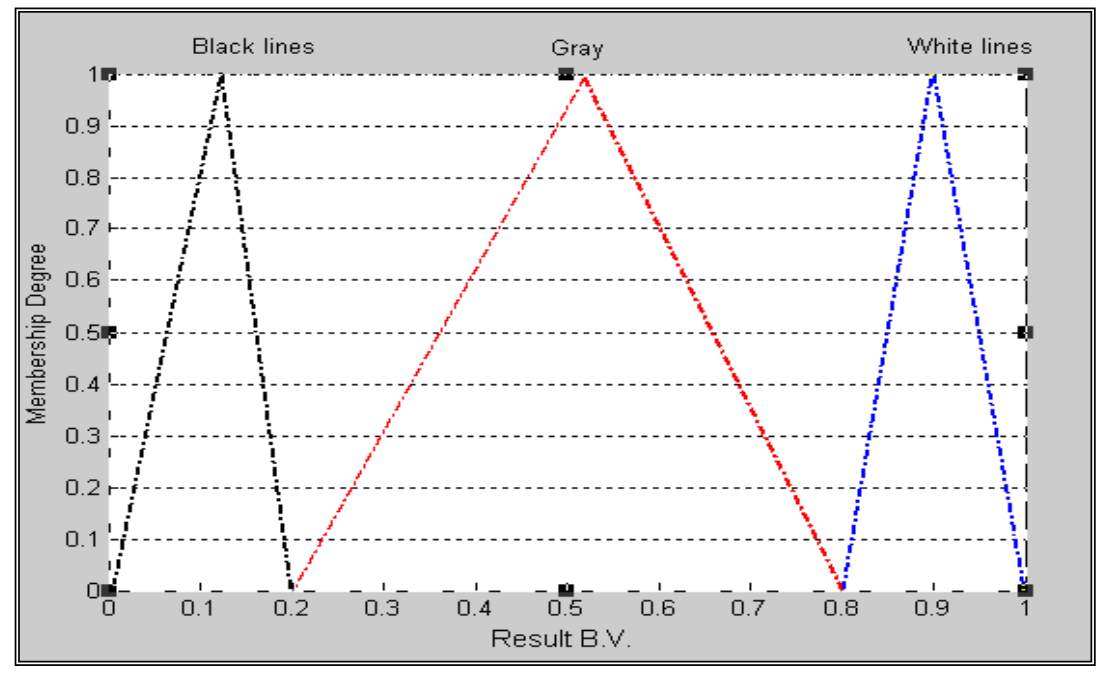

الثكل(6) دوال العضوية للاخراج (ResultBV)

نلاحظ من الثكل السابق ان الاخراج (ResultBV) يتكون من 3 دوال عضوية (3 Membership Functions) تمثل قيم التوهج الواطئة (Low Brightness Value) التي بدورها تولد خطوطاً سوداء، اما الدالة الثانية وهي (Gray) التي تكون محصورة بين (0.8-0.2) فتمثل التدرجات الرمادية للصورة الفضائية اي المساحات الواسعة للصورة الفضائية كالصحاري والبحار والمحيطات، واخيراً الدالة Hi الثالثة (White lines) التي تكون محصورة بين (1-0.8) وتمثل قيم التوهج العالية Brightness Value والتدرجات الرمادية يولد الحافات في الصورة الفضائية ومن ثم يتم تقليل التشويش (noise) وزيادة تفاصيل الصورة الفضائية.

Exit 10.7 ويمكن توضيح معالم بعض الصور الفضائية واجراء التحسين عليها من خلال الصور الآتية: 

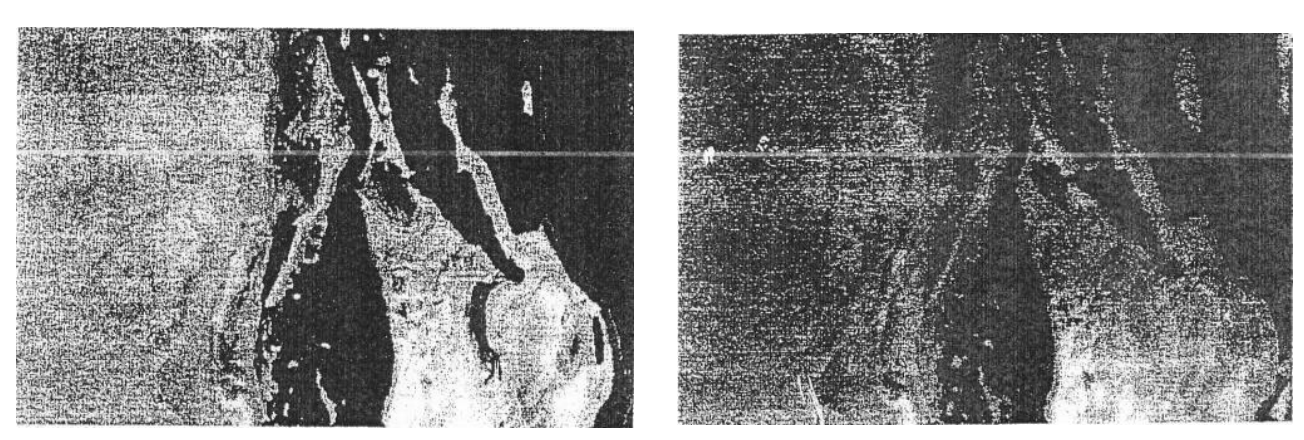

صورة فضائية لمنطقة البحر الأحمر بعد اجراء عملية التحسين

صورة فضائية لمنطقة البحر الأحمر قبل

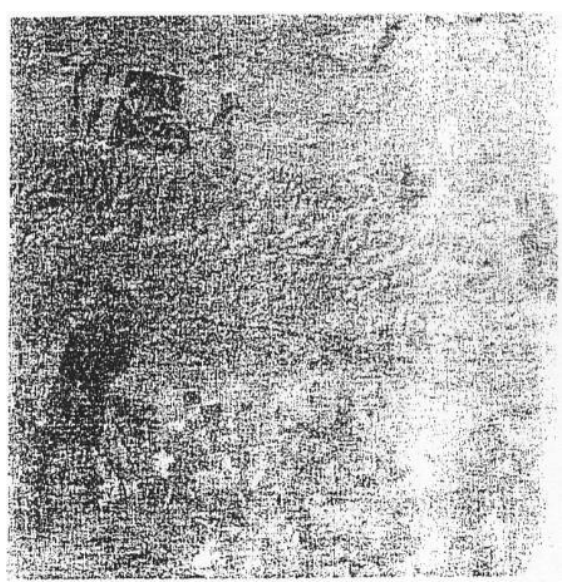

صورة فضائية لمنطقة جبل شيخ إبراهيم قبل اجراء عملية التحسين 
لقد أسفر التطبيق الفعلي للنظام عن الحقائق الآتية :

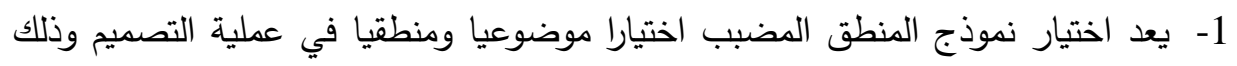

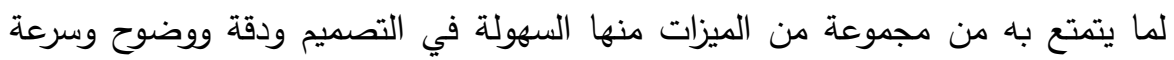
فائقة في تقييم الاداء. 2- أظهرت المعالجة ألحاسوبية لمدخلات النظام من خلاد سلسلة القواعد المعتمدة لكل متغير من متغيراتها ، إمكانية استثمار النموذج في تفسير معالم الصورة الفضائية وتحديد الحافات والتدرجات الرمادية والى غير ذلك من المعلومات.

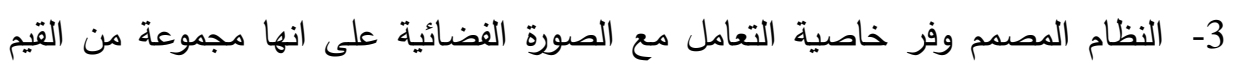
الرقمية ومن ثم يمكن اجراء اي تغيير على هذه القيم والتي تؤدي الى اجراء تغيير في معالم الصورة. 4- هنالك الكثير من البرامجيات الحاسوبية الحديثة التي من خلالها يمكننا استغلال الحاسوب

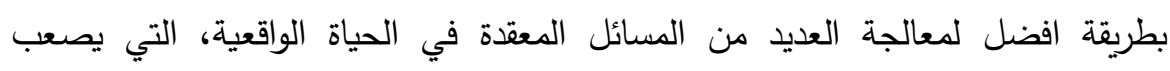

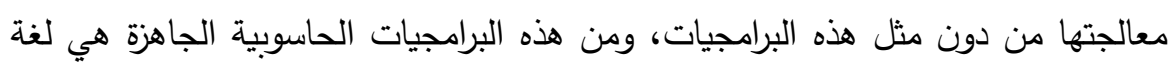
MATLAB والتي يمكن من خلالها تصميم نماذج مضبية.

1- ضرورة اجراء دراسات اخرى باتجاهات مختلفة، وفي العديد من التطبيقات في الحياة

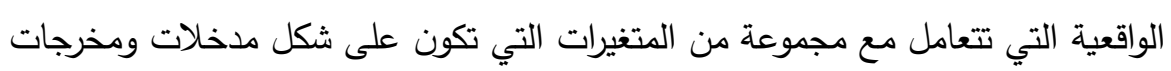
والتي تمثل بالتالي هيكل النظام بشكل عام.

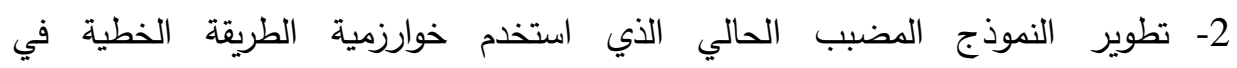
تحسين حافات صور الاقمار الصناعية عن طريق تصميم نموذج مضبب لعملية الالتفاف

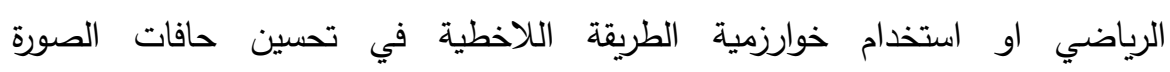
الفضائية.....الخ من الخوارزميات. 3- يمكن بناء نظام ذكي لتطبيق خوارزميات تحسين حافات صور الاقمار الصناعية كأن

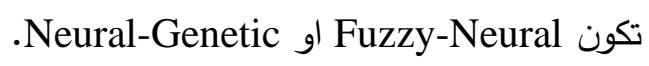




\section{المصادز.}

شيريل بليرين،(2007) "الصور التي يلتقطها القر الصناعي landsat للكرة الارضية"،

http://usinfo.state.gov/xarchives/display.html?p=washfile-

arabic $\& y=2007 \& m=$ April $\& x=20070411122037$ liameruoy 0.5984766

الدباغ، سفيان سالم (2003):"تصميم نظام حاسوبي لتقدير قوة مقاومة انضغاط الأسمنت

البورتلندي"، رسالة ماجستير، كلية علوم الحاسبات والرياضيات، جامعة الموصل.

[3] Konar A., ( 2000 ), "Artificial Intelligence and Soft Computing", Behavioral and Cognitive Modeling of the Human Brain, dept. Electronics and Tele-Communication Engineering, University of Jadvpur, Calcutta, India, CRC Press, Inc., PP.47. http"//homepages.cae.edu/ece533/project/enhancement.html

[4] Hellmann M.,(2001)"Satellite Image Enhancement",project, http://www.arabien.net/arabiaall/2000/12.html

[5] Turksen B.,Bilgic T.,(1995),"Measurment of Membership finctions, Theoritical and Impirical work", Fuzzy Sets and Systems, http://www.ie.boun.edu.tr/ taner/publications/papers/membership.pdf

[6] Becerra V.M. (1995): "Advanced system identification", lecture1, http://www.personal.rdg.ac.uk/ shs99vmb/notes/asi/Lecture1.pdf

[7] Astrom \& Wittenmark (1997) " Computer Controlled SystemsTheory and Design", Third Edition, Pentice Hall, Inc., London.

[8] Rao, B.V. (1998): "C++ Neural Network and Fuzzy Logic", $2^{\text {nd }}$ edition, MIS Press.

[9] Zimmermann, H.J.,(1987),'Fuzzy set design making \& Expert systems, Boston, Kluwer .

[10] Mathworks, The mathworks Inc. (2002): "MATLAB The Language of Technical Computing", Version 6.5, Release 13, June 18. 